\title{
Aus den Sitzungen.
}

Sitzung vom 16. X. 11. - Beginn $9 \frac{1}{2}$ Uhr. Die Sitzung leitet der Vorsitzende Gr ün berg. - Das Protokoll der Sitzung vom 9. X. 11 wird verlesen und genehmigt. - Der Vorsitzende macht der Gesellschaft die Mitteilung, dafs Herr Prof. Dr. H. Koshewnikow in Moskau am 19. IX. sein 25jähriges Jubiläum als Forscher auf dem Gebiete der Zoologie begeht. Die Gesellschaft beschliefst, dem Jubilar durch den Vorstand ihre Glückwünsche zu senden. - Grünberg schlägt ferner als zukünftiges neues Mitglied Herrn F. S chumacher, Lehrer, Kagel bei Rehfelde, vor. - Ohaus referiert aus der Arbeit von $\mathrm{Hein}$ rich Prell, Marburg, „Biologische Beobachtungen an Termiten und Ameisen ", über einen Termitenraubzug von Megaloponera, welchen die Mitglieder der akademischen Studienfahrt nach Ost-Afrika 1910 in der Gegend von Njussi bei Amani beobachten konnten. Der genannte Zug hatte eine Länge von $1^{1} / 2$ m bei einer Breite von mehr als $10 \mathrm{~cm}$. Ein grofser Teil der Individuen dieses Ameisenzuges war mit Kadavern geraubter Termiten beladen. Sehr merkwürdig am ganzen Zuge war das laute Zirpen, das die Ameisen ununterbrochen von sich gaben. An einer anderen Stelle bei Moschi in der Nähe des Kilimandjaro konnten sehr interessante Beobachtungen an bei Termiten lebenden Carabicidenlarven gemacht werden. Diese Larven, zu Glyptus punctulatus Chaudoir gehörig, wurden mehrfach in verschiedenen Stadien bei einer kleinen Termitenart (Microtermes incertus) gefunden. Kuntzen bemerkt hierzu, dafs Vertreter anderer Carabicidengruppen, z. B. einiger Chlaeniinen, vermutlich in Termitennestern leben dürften. - $\mathrm{Ohaus}$ legt eine interessante neue Melolonthide, auf welche er eine neue Untergattung des Genus Ceraspis gründet, aus San Antonio do Iça am oberen Amazonas vor; er nennt sie Isoceraspis Duckei zu Ehren des Sammlers A. Ducke, des bekannten Hymenopterologen am Museum in Para. Im Anschlufs an das Referat von Ohaus bemerkt $\mathrm{Kuntzen}$ einiges über die systematische Stellung und die Merkwürdigkeiten der Carabidengattungen Orthogonius und Glyptus, die im Königlichen Museum fast vollständig vertreten seien, und unter denen sich noch vieles Neue findet. Sodann zeigt $\mathrm{Kuntzen}$ Broscosoma baldense (Rosenhauersche Cotypen), Miscodera arctica (aus dem Brieselang und dem Nauener Stadtforst bei Berlin, aus der Stettiner Umgegend, aus Wyborg, aus Lappland) und ein typisches Stück der Form erythropus Motsch., das in den Besitz von L. W. Schaufufs, und mit dessen Sammlung an das Königliche Museum gekommen ist, ferner die prachtvolle grüne Broscosoma ribbei Putz. 
aus Darjeeling. Im Anschlufs daran machte er aufmerksam auf die interessante Verbreitung der Gattung Miscodera (Mittel-Europa, Nordisches Europa, das ganze nördliche Sibirien und nördliches Nord-Amerika), der Gattung Broscosoma (Monte Baldo [baldense], Himalaya [ribbei], Jünnan und Japan) und der gesamten Brosciden (Paläarktisches Gebiet, nördliches nearktisches Gebiet, Chile, Argentinien, Paraguay, Süd-Brasilien und vor allem je fast gleich artenreich Australien und Neu-Seeland). O haus und $\mathrm{R}$ e i n e ck teilen ihre Beobachtungen über die Lebensweise und den Fang von Broscosoma baldense auf dem Monte Baldo mit. — Grünberg bespricht die Arbeit von Willy Ramme (Charlottenburg) „Ein Beitrag zur Kenntnis der Orthopterenfauna der Mark Brandenburg". - Ferner demonstriert Grünberg eine Anzahl Vertreter der indo-australischen Noctuiden-Gattung Apsarasa Moore, die durch ihre ungewöhnlich bizarr schwarz und gelb gezeichneten Vorderflügel auffallen. Bis zum Erscheinen des neunten Bandes von Hampsons Lepidoptera Phalaene vor etwa kurz einem Jahre waren nur 2 Arten bekannt, varians Westw. und figurata Moore. Hampson beschrieb in dem genannten Werk 2 neue Arten. Unter den Beständen des Berliner Zoolog. Museums fanden sich noch weitere 3 Arten, so dafs die Anzahl der bekannten Arten jetzt auf 7 gebracht ist, welche in der hiesigen Museumssammlung sämtlich vertreten sind. - Schlufs $11 \mathrm{Uhr}$.

Sitzung vom 23. X. 11. - Um 8 Uhr findet eine Vorstandssitzung statt. - Die vom stellvertretenden Vorsitzenden Gre in e r geleitete Sitzung wird um $9^{1} / 2$ Uhr eröffnet. - Das Protokoll der Sitzung vom 16. X. 11 wird verlesen und genehmigt. Als Gast wohnt Herr E. Heidenreich (Dessau) der Sitzung bei. - Herr Heinrich Prell, Marburg a. d. Lahn, Biegenstrafse $31 \mathrm{II}$, wird von Dr. Ohaus als Mitglied unserer Gesellschaft vorgeschlagen. - O haus spricht über die systematische Stellung und über den Bau der Mundteile von Anatista Lafertei. Die Art wurde von Brème als Melolonthide beschrieben und von Lacordaire an die Grenze zwischen Melolonthiden und Ruteliden gestellt. Wie der Vortragende schon früher (Stett. E. Z. 1905) ausgeführt hat, ist sie eine Rutelide und gehört zur Gruppe der Spodochlamiiden, einer der Abteilungen der Ruteliden mit vertical stehender Oberlippe. In dieser Abteilung, zu der aufserdem die Anoplognathiden, Geniatiden und Adoretiden gehören, nehmen sie eine besondere Stellung dadurch ein, dafs den Lippen ein mittlerer Fortsatz oder Vorsprung fehlt, so dafs die beiden Kiefer der beiden Seiten einander in der Mitte be- 
rühren können, während bei den 3 anderen Untergruppen die Mundöffnung durch einen mittleren Vorsprung der Lippen in eine linke und eine rechte geteilt ist, die jede für sich allein zum Beilsen benutzt werden. Ganz eigenartig ist der Bau der Oberkiefer bei den Spodochlamiiden. Hier trägt der basale Mahlzahn an seinem Vorderrand mehrere grofse runde Öffnungen, die gewöhnlich durch eine Falte der dicht behaarten Pars membranacea zwischen Mahlzahn und Schneide resp. Spitzenteil verdeckt werden - die Öffnungen glattwandiger Kanäle, die in den Raum hinter dem Mahlzahn führen und weiterhin auf die Dorsalseite der Oberkiefer münden. Da über die Lebensweise der Spodochlamiiden absolut nichts bekannt ist, lälst sich über den Zweck dieser Bildung auch nichts sagen. Ferner übermittelt er Grüfse von unserem Mitglied Schilsky an die Gesellschaft. - Schlufs 11 Uhr.

Sitzung vom 30. X. 11. - Um 8 Uhr findet eine V o r standssitzung statt, die sich mit den Vorbereitungen für die event. auf den 11. XII. 11 anzuberaumende Generalversammlung beschäftigt. - Beginn der Gesamtsitzung $9^{1} / 4$ Uhr. Leiter der Verhandlungen: Erster Vorsitzender Gr ünberg. - Das Protokoll der letzten Sitzung wird verlesen und genehmigt. - Der Vorsitzende führt sodann aus, dafs die Versammlung eine wichtige Angelegenheit zu beraten habe, nämlich den Antrag, der zur Anbahnung einer Vereinigung mit dem "Berliner Entomologis chen Verein" der Generalversammlung zur Beschlufsfassung vorzulegen sei. Er bitte zunächst $\mathrm{Ohaus,} \mathrm{der} \mathrm{Vor-}$ sitzender der aus Mitgliedern beider Vereine zusammengesetzten Kommission gewesen sei, über die Vorgeschichte der Angelegenheit $\mathrm{zu}$ berichten. - $\mathrm{Ohaus}$ schildert darauf die Entwicklung des Planes. Er führt aus, dafs die Anregung zu der Vereinigung von der „Berliner Entomologischen Gesellschaft“ ausgegangen sei, dafs dann eine Zusammenkunft von Vorstandsund andern Mitgliedern beider Vereine im "Heidelberger" stattgefunden habe, und dafs der Beschlufs, den diese Versammlung gefafst, dahin verwirklicht worden wäre, dafs beide Vereine Vertreter für die gemischte Kommission zur weitern Vorbereitung der Vereinigung gewählt hätten. Die gemischte Kommission habe eine Reihe von Sitzungen abgehalten. Sie habe einen Statutenentwurf durchberaten und sich dahin geeinigt, dafs nach der Annahme des Statutenentwurfs durch die beiderseitigen Generalversammlungen der "Berliner Entomologische Verein" in Liquidation treten solle. Später habe sich dann aber herausgestellt, dafs die Herren des andern Vereins sich zu wenig vorher mit dem Plenum 
ins Einvernehmen gesetzt hätten. Das Plenum habe eine gewisse Probezeit zur Herbeiführung näherer Beziehungen gefordert. So sei dann schliefslich in der gemischten Kommission ein Antrag angenommen worden, der beiden Vereinen zanächst die Abhaltung von 2 gemeinschaftlichen Sitzungen in jedem Monat vorschlägt. Der Beschlufs der gemischten Kommission, der alsbald von G r ünberg verlesen wird, hat folgenden Wortlaut: „Die Vereine halten vorläufig eine Anzahl gemeinsamer Sitzungen ab, in der Art, dafs jeden Monat eine Sitzung bei der "Deutschen Entomologischen Gesellschaft", eine zweite im "Berliner Entomologischen Verein" im Lokal und unter Leitung des betreffenden Vereines stattfindet. Den Modus dieser Sitzungen vereinbaren die beiderseitigen Vorstände. Dieser Beschlufs ist den baldigst einzuberufenden Generalversammlungen vorzuschlagen". - P a p e kann den Ausführungen von $\mathrm{O} h$ a u s nicht beipflichten. $\mathrm{O}$ haus habe gesagt, der andere Verein sei an die „D. E. G.“ herangetreten mit der Frage, ob sie einer Vereinigung geneigt sei. Nun aber kämen die andern Herren und sagten, erst müssen wir eine Probezeit haben. Er, P a p e, fände das sonderbar. Er sei von dem. Standpunkt eines Gegners der Vereinigung, den er anfangs eingenommen, zurückgetreten, müsse sich ihm aber jetzt wieder zuwenden und bäte die Anwesenden, es sich zweimal zu überlegen, ob dem Antrag der gemischten Kommission zuzustimmen sei. - Greiner stellt zu den Ausführungen von Pape zuerst richtig, dafs die Anregung zu der Vereinigung nicht vom „Berliner Entomologischen Verein" als solchem, sondern nur vom Vorstande ausgegangen war. Bei einem gleichen Verhalten des Vorstandes würde man auch in der „D. E. G.“ gesagt haben, dazu habe der Vorstand kein Recht gehabt. Er, Greiner, glaube, dafs so allein die Stimmung erklärt werden müsse, die schliefslich im "Berliner Entomologischen Verein" mafsgebend geworden sei. Die grofse Majorität des Berliner Vereins wolle jedenfalls die Vereinigung. Der Widerspruch, den der zuerst dazu vorgeschlagene Weg gefunden, sei menschlich begreiflich und dürfe die „D. E. G." nicht abhalten, dem Plan, eine grofse entomologische Gesellschaft zu begründen, ihre Zustimmung zu geben. Wenn man den Gedanken als gut erkannt habe, solle man doch nicht um kleinlicher Bedenken willen der Sache Schwierigkeiten in den Weg legen. Die wöchentlichen Sitzungen der „D. E. G.“ blieben ja wie bisher bestehen, nur würde die „D. E. G." allmonatlich an einer Sitzung des "Berliner Entomologischen Vereins", und umgekehrt der "Berliner Entomologische Verein" an einer Sitzung der „D. E. G." teilnehmen. Er, Redner; bäte, ehe beide Vereine sich kennen gelernt, dem gewifs freudig zu begrüfsenden 
Plan weiter keine Schwierigkeiten in den Weg zu legen. $\mathrm{Ohaus}$ unterstützt durch weitere Erklärungen die Ausführungen von Greiner. - Pape hält demgegenüber seinen Widerspruch aufrecht. - Schallehn kann sich dem, was Pape gesagt hat, so ganz nicht. verschliefsen. Zum Kennenlernen seien doch andere Wege vorhanden. Es gäbe doch in beiden Vereinen Herren, die hüben wie drüben Mitglieder wären. Er, Redner, habe sich auch sagen lassen, dals der "Berliner Entomologische Verein" ein Lepidopterologenverein sei. Nun habe man ja vor kurzem in der „D. E. G.“ eine Sezession erlebt. Es frage sich daher, ob nicht bei Abhaltung gemeinschaftlicher Sitzungen mit dem „Berliner Entomologischen Verein" die Herren, die sich dadurch in ihren Interessen als Coleopterologen bedroht fühlten, lieber zur Sezession abschwenken würden. Dort solle ja, wie man höre, die Coleopterologie in reinstem Mafse gepflegt werden. Bei der Gelegenheit möchte er, Schallehn, sich noch eine Anregung gestatten. Die "D. E. G." habe, nachdem nunmehr die Scheidung vollzogen, mit dem "Deutschen Entomologischen National-M useum" nichts mehr zu tun. Es lägen deshalb auch keine Gründe mehr vor, dafs sich die Herren mit den übrigen Vereinsmitgliedern nicht wieder zusammenschlössen. Nach seinem Dafürhalten würde es sich unter diesen Umständen fragen, ob nicht seitens des Vorstandes ein Schreiben an den Führer der Sezession mit der Aufforderung abgehen könnte, dafs die Herren, da jetzt alles geregelt sei, doch wieder regelmäfsig an den Sitzungen teilnehmen sollten. - $\mathrm{O}$ h a u s befürwortet nochmals die Annahme des Kommissionsantrages, der doch wenigstens den Weg weise, $\mathrm{zu}$ einer Verständigung und auch zu einer Vereinigung zu kommen. Grünberg betont gegenüber den Ausführungen Schallehns, dafs weder der „Berliner Entomologische Verein“ ein Lepidopterologen-, noch die „D. E. G. “ ein Coleopterologen-Verein sei. Beide Vereine seien vielmehr universell entomologische Gesellschaften. Ein Schreiben an Herrn Dr. Hor $\mathrm{n}$ und die anderen Herren zu richten, die den Versammlungen fernblieben, müsse er, Gr ü nbe r g, entschieden ablehnen. Die Herren seien ja noch Mitglieder und es stände ihnen deshalb frei, so oft zu kommen, wie sie wollten. Von einer besonderen Aufforderung könne unter keinen Umständen die Rede sein. - Nach weiteren Bemerkungen von Pape und Ohaus nimmt Soldanski das Wort, um ebenfalls für den Kommissionsantrag einzutreten. Gegenüber $\mathrm{Sch}$ alle hn weist auch Soldanski den Vorschlag, ein Schreiben an die sogenannte Sezession zu richten, ganz energisch zurück. - Höfig kann Pape nicht ganz unrecht geben. Insbesondere vertritt er die Ansicht, dafs man sich in der vorgeschlagenen Weise auch 
noch nicht nach 2 oder 3 Jahren kennen gelernt haben würde. An der weiteren Erörterung beteiligen sich Pape, der Vorsitzende und Ohaus. - Schliefslich ergreift noch Ahlwa $\mathrm{rth}$ das Wort, um Pape aufzufordern, die Sache selbst doch höher zu stellen als kleinliche Bedenken. - Der Vorsitzende bringt dann den Antrag der Kommission in einer vom Vorstande beschlossenen Fassung zur Verlesung. Der Antrag lautet so folgendermafsen: „Die ,Deutsche Entomologische Gesellschaft' und der, Berliner Entomologische Vere i n' halten von Beginn des Jahres 1912 zur Anbahnung näherer Beziehungen und zwecks späterer Verschmelzung monatlich 2 Sitzungen gemeinsam ab, die erste bei der, Deutschen Entomologischen Gesellschaft, die zweite bei dem ,Berliner Entomologischen Verein'. Über die Art der Durchführung sollen sich zunächst die beiderseitigen Vorstände ins Einvernehmen setzen. Die Regelmälsigkeit der Versammlungen der Gesellschaft werden hierdurch nicht berührt." - Der Antrag zirkuliert darauf unter den Anwesenden und findet alsbald 24 Unterschriften. Der Vorsitzende schlägt vor, zur Beschlufsfassung über den Antrag eine Generalversammlung auf den 11. XII. 11 einzuberufen. Der Vorschlag wird mit grofser Majorität angenommen. - H e y n e zeigt darauf in schönen Exemplaren Phalacrognathus mülleri M. L. und erwähnt dabei, dafs die ersten Paare dieses Tieres, die vor 25 Jahren aus Nord-Australien nach Europa gekommen seien, $300 \mathrm{Mk}$., die weiteren $270 \mathrm{Mk}$. gekostet hätten. Heute sei der Preis bedeutend gesunken, obwohl das Tier noch immer zu den Seltenheiten zähle. Heyne lälst ferner neue Literatur zirkulieren, so den Lepidopteren-Katalog (I-Ia), C a lvers Käferbuch, Lfg. 19 , Heft 8 der „Mis cellanea entomologica“, Nr. 322 der "Revue Linnéenne" und 5 Lieferungen des Seitz schen Schmetterlingswerkes (I, 88 und II, 101-4). - Pape beklagt, dafs so wenig aus den Vorstandssitzungen mitgeteilt würde. So habe er gehört, dafs die Schubertsche Bibliothek für die Gesellschaft angekauft sei. Offiziell sei aber bisher der Versammlung nichts darüber berichtet worden. - Kuhnt erwidert, dafs der Ankauf der Schubertschen Bibliothek aus Mitteln bewirkt sei, die Moser der Gesellschaft zur Verfügung gestellt habe. Deshalb habe man in den Versammlungen auch nicht weiter darüber gesprochen. - Pape nimmt diese Mitteilung zum Anlals, um die Anwesenden aufzufordern, ihr Glas auf das Wohl des hochherzigen Geschenkgebers zu leeren. - Schlufs 11 Uhr. 
Sitzung vom 6. XI. 11. - Beginn 91/2 Uhr. Leiter der Verhandlungen : Erster Vorsitzender G r ü n b e r g. - Das Protokoll der vorigen Sitzung wird verlesen und genehmigt. Der Vorstand des "Berliner Entomologischen Vereins" teilt in einem Schreiben mit, dafs das Plenum dem Antrag des Vorstandes, vom nächsten Jahre ab eine Anzahl gemeinsamer Sitzungen mit der „Deutschen Entomologischen Gesellschaft“ abzuhalten, mit grofser Majorität zugestimmt habe. Der Vorsitzende verliest das Schreiben und bemerkt dazu, er hätte gewünscht, es schon am vorigen Montag vorlegen zu können; es sei aber leider erst am Dienstag in seine Hände gelangt. Im übrigen könnten die Mitglieder der „Deutschen Entomologischen Gesellschaft" aus dem Schreiben ersehen, dafs der Vorstand des Berliner Vereins sich genau an den Wortlaut des Kommissionsbeschlusses gehalten habe. Der Vorstand der „Deutschen Entomologischen Gesellschaft" habe eine etwas andere, den Sinn jedoch nicht berührende Form gewählt, weil er den Antrag in prägnanter und präziser Fassung vor der Generalversammlung zur Kenntnis der Mitglieder bringen müsse. - $\mathrm{O}$ ha us teilt mit, dafs er das Novemberheft der Zeitschrift leider noch nicht verteilen könne, weil nach einer Angabe der Druckerei Korrekturen verloren gegangen seien. Das Heft würde erst am nächsten Montag verteilt werden. Die Anträge zur Generalversammlung kämen auch dann noch rechtzeitig in die Hände der Mitglieder. - Kuhnt berichtet über die neueste Literatur und beantragt im Anschlufs daran die Anknüpfung eines Zeitschriftenaustausches mit dem „Bulletin de la Société Entomologiqued'Égypte“. Der Antrag wird mit grofser Majorität genehmigt. - Unter der neu eingegangenen Literatur befindet sich auch eine Probenummer von K. B eifswanger: "Im Lande der heiligen Seen. Reisebilder aus der Heimat der Chibcha-Indianer (Columbien)“, das den Mitgliedern der Deutschen Entomologischen Gesellschaft für den ermälsigten Preis von $25 \mathrm{Pf}$. pro Lieferung angeboten wird (das ganze Buch soll 12 Lieferungen nicht überschreiten). O ha us macht auf die dem Hefte beigegebenen, vorzüglich reproduzierten Photographien aufmerksam. Gleich das erste Bild, Indianische Salzhändler in Guatavitá, ist ungemein charakteristisch, stellt es doch eine Szene dar, die sich im ganzen interandinen Gebiet beobachten läfst. Das fast ganz reine Steinsalz wird in Peru in leichtem Bergbau gewonnen, an Ort und Stelle in Form kleiner Ziegelsteine zurechtgehauen und bildet dann im ganzen Kordillerengebiet einen der wichtigsten Handelsartikel. Bis weit nach Osten an den Nebenflüssen des Amazonas trifft man diese Salzsteine und kann oft beobachten, wie der Besitzer, nachdem er seinen Braten 
oder Fisch damit gerieben oder geklopft, seinen kostbaren Stein sorgfältig ableckt, in ein grofses Blatt wickelt und ihn dann neben dem Feuer trocknet. Auffallend sei es dabei, wie besonders zahlreiche Dipteren und Hymenopteren durch das Salz angezogen würden. Die Bilder: „Unser Lasttier im Morast" und "Schlechte Wegeverhältnisse" geben einen Begriff von den Beschwerden, mit denen das Reisen im Andengebiet verbunden ist. Die sogenannten Maultierleitern, auf der Ostseite Südamerikas pilões, Mörser, genannt, stellen eines der schwersten Hindernisse beim Reisen mit Maultieren dar; sie entstehen durch die Gewohnheit der Maultiere, stets in die Fufsstapfen ihrer Vorgänger zu treten, wodurch allmählich in dem weichen Lehmboden tiefe Löcher ausgetreten werden, die sich mit Wasser füllen und in denen die Tiere beim Stolpern leicht ein Bein brechen. An dem Bild: „Leiter und Webstuhl aus einer Indianerhütte bei Bolivar" ist u. a. die Wand des Hauses beachtenswert. Sie zeigt, wie der Lehm, mit dem das Flechtwerk zwischen den Pfosten überstrichen wird, beim Trocknen sich von dem Flechtwerk löst und überall rissig wird. Diese Risse sind bald die Schlupfwinkel von allem möglichen Ungeziefer, Wanzen und Milben, Zecken und Kakerlaken (Blattiden), auch Skorpionen und Spinnen, die auf dieses Ungeziefer und die nicht weniger zahlreichen Fliegen Jagd machen. Gr ü $\mathrm{nberg}$ bemerkt zu dem Referat von $\mathrm{Ohaus}$, auch bei uns könne man sehen, dafs Salz Insekten anzöge, denn eben deshalb seien sie auf den menschlichen Schweifs versessen. Besonders an heifsen Tagen werde man häufig im Walde von A n thomyiden verfolgt, die keineswegs zu den blutsaugenden Dipteren gehörten, die vielmehr lediglich der Schweifsgeruch anlocke. - Grünb e r g referiert weiter über eine anatomische Arbeit von $\mathrm{Rich}$ ard Vogel, Über die Innervierung der Schmetterlingsflügel und über den Bau und die Verbreitung der Sinnesorgane auf denselben. Zeitschr. f. wissensch. Zool., Bd. 98, Heft 1, 1911. Die Nervenäste verhalten sich in ihrem Verlauf im Vorder- und Hinterflügel wesentlich gleich. Der jeden Flügel innervierende Nervenstamm teilt sich dicht vor der Flügelwurzel in 3 Äste, einen vorderen, einen mittleren und einen hinteren, welche getrennt in den Flügel eintreten, um sich bald darauf in die einzelnen, dem Verlauf der Flügeladern folgenden Äste zu teilen. Feine Verästelungen der Adernerven, welche in die Flügelfläche eintreten und hier einzelne zerstreute Sinneszellen innervieren, kommen ebenfalls vor, besonders an der Flügelspitze, wo die Verwachsung der beiden Flügellamellen eine weniger enge ist. Die eigentlichen Sinnesorgane der Schmetterlingsflügel, auf deren feineren histologischen Bau hier nicht näher eingegangen werden soll, sind nun ver- 
schiedener Art. Man unterscheidet Sinneskuppeln, innervierte Schuppen, Randader-Sinneshärchen und schliefslich Chordotonalorgane. Die Sinneskuppeln treten einzeln oder gruppenweise auf, besonders wichtig sind 2 dicht an der Flügelwurzel in den Verlauf der beiden ersten Nervenstämme vor deren Gabelung eingeschaltete Gruppen, welche der Verfasser als Costal- und Subcostalgruppe bezeichnet. Die in diesen Gruppen vereinigten Sinneszellen ordnen sich wieder in verschiedene Untergruppen, und es ist auffällig, wie bei den Familien, Gattungen und selbst Arten diese Gruppierung in enger Anlehnung an die morphologischen und systematischen Merkmale wechselt und so eine sehr wertvolle Ergänzung zur Beurteilung der letzteren wie der phylogenetischen und verwandtschaftlichen Beziehungen liefert. Zahlreiche einzelne Sinneskuppeln sind längs der Adern und des Flügelrandes angeordnet. - Die innervierten Schuppen, von schmal lanzettlicher Form mit lang ausgezogener feiner Spitze, kommen bei allen Schmetterlingen ohne Ausnahme vor, bei den primitivsten wie bei den höchst differenzierten Formen, und besitzen daher zweifellos ein sehr hohes phylogenetisches Alter, sind vielleicht die ältesten Schuppen überhaupt. Da sie meist auf den Adern, besonders auf der Randader stehen, so neigt Verfasser zu der Meinung, dafs die Beschuppung der Flügel einst von den Adern aus ihren Anfang genommen habe, eine Annahme, die in analogen Befunden bei anderen Insektenordnungen, z. B. bei den schuppentragenden Dipteren, eine wesentliche Stütze findet. - Die Randader-Sinneshärchen stehen immer auf der Unterseite der Randader. Sie sind bei den Rhopaloceren im Hinterflügel häufiger als im Vorderflügel. - Die Chordotonalorgane, über deren Bau und Lage der Verfasser sich noch nähere Mitteilungen vorbehält, finden sich an der Wurzel des Vorder- und Hinterflügels und werden von Abzweigungen des zweiten Nervenstammes versorgt; nur im Vorderflügel findet sich noch ein weiteres, vom dritten Stamm innerviertes Organ. - Über die Funktion der Sinnesorgane des Schmetterlingsflügels äufsert sich Verfasser nur vermutungsweise. Die Sinnesschuppen und die Randader-Sinneshärchen hält er für Tastorgane, welche vielleicht zur Wahrnehmung der Luftbewegung dienen. Sehr schwierig ist eine Deutung der Sinneskuppeln. Verfasser ist der Ansicht, dals sie für den Flug von wesentlicher Bedeutung sind, kann aber über eine nähere Funktion keine Vermutung aufstellen. Die Chordotonalorgane hält er für auf dem Prinzip des Zuges basierende statische Organe und glaubt, dals sich aus solchen Organen erst die „tympanalen Chordotonalorgane" entwickelt haben. - Im Anschlufs an die Ausführungen G r ün be r g s bemerkt $\mathrm{O}$ ha us, dafs auch bei den Käfern, speziell 
bei den Lamellicorniern, die Adern der Deck- oder Vorderflügel häufig mit Tastorganen, sogenannten taktilen Borsten, besetzt sind. Diese sitzen inmitten fein umwallter Punkte auf einem Höckerchen. Auch da, wo die ganzen Flügel fein behaart sind, stehen häufig auf den Adern oder Rippen solche taktile Borsten, stets länger, häufig auch anders gefärbt als die umgebenden Haare; seltener stehen sie in den Punkten, die beiderseits die Adern oder Rippen begrenzen, in den sogenannten primären Punktreihen. - Schlufs 11 Uhr.

Sitzung vom 13. XI. 11. - Beginn 9 $\frac{1}{2}$ Uhr. Leiter der Verhandlungen: Stellvertretender Vorsitzender Greiner. - Das Protokoll der vorigen Sitzung wird genehmigt. - O ha us legt das neu erschienene Heft der „Deutschen Entomologis chen Zeitschrift" vor, bespricht den Inhalt und erklärt nochmals das verspätete Erscheinen. Er bemerkt dabei, dafs auch für das nächste Heft, wie überhaupt für den kommenden Jahrgang, bereits reichlich Manuskript vorhanden sei. Ferner macht $\mathrm{O}$ ha us nochmals auf das schon im vorigen Sitzungsbericht erwähnte Lieferungswerk von Beifswanger: "Im Lande der heiligen Seen, Reisebilder aus der Heimat der Chibcha-Indianer" aufmerksam. In Zürich ist ein entomologischer Verein gegründet worden. Schulthefs und Standfufs haben dies zum Anlafs genommen, um am Abend der Vereinsgründung der „Deutschen Entomologischen Gesellschaft" in einer an Mos e r gerichteten Karte herzliche Grüfse zu übermitteln. Auf Vorschlag von $\mathrm{O}$ ha u s wird beschlossen, die Grülse der Herren mit bestem Dank für das freundliche Gedenken in gleicher Weise zu erwidern. -- G r ünberg teilt mit, dafs das Kgl. Zoologische Museum in Berlin die Staphylinidensammlung Schuberts aus dessen Nachlafs erworben hat. Ferner hat das Kgl. Museum die Ithomiidensammlung von $\mathrm{H}$ aensch angekauft. Letztere Sammlung ist, wie Grünberg hervorhebt, aufserordentlich wertvoll und zuletzt von ihrem Besitzer zur Bearbeitung der Ithomiiden für das Seit z sche Werk benutzt worden. - $\mathrm{O}$ ha u s legt die bekannten Arten der Gattung Aclopus Er. vor, sowie eine neue Art, die von Herrn Wünsche im Staate São Paulo in Brasilien gesammelt wurde und demnächst in dieser Zeitschrift beschrieben werden soll. Die systematische Stellung dieser Gattung, über die sich der Vortragende in unserer Zeitschrift 1909 , p. 426 u. f., ausführlich geäufsert hat, wird kurz besprochen und auch die Arrowsche Auffassung von der Stellung dieser Gattung (Transactions of the London Entomolog. Soc. 1909, p. 485) dargelegt. - Schlufs 11 Uhr. 
Sitzung vom 20. XI. 11. - Beginn $9^{1} / 2$ Uhr. Leiter der Verhandlungen: Erster Vorsitzender Grünberg. - Das Protokoll der vorigen Sitzung wird verlesen und genehmigt. - Die Herren Hedicke, F. Schumacher und Hein rich Prell werden, nachdem die statutengemäfse Veröffentlichung ihrer Namen im letzten Heft der Zeitschrift erfolgt ist, einstimmig als Mitglieder aufgenommen. Als neues Mitglied wird sodann von $\mathrm{O}$ ha u s Herr stud. phil. C. R hode, Münster i. W., Herrmannstr. 21, in Vorschlag gebracht. - Ein auswärtiges Mitglied, Herr Wilhelm $\mathrm{N}$ i e pelt, hat seine Photographie für das Album der Gesellschaft übersandt. - Die bekannte entomologische Buchhandlung von W. J unk, Berlin W, hat für einen Katalog, den sie demnächst herausgeben wird, der D. E. G. die unentgeltliche Aufnahme eines Inserats angeboten. Die Versammlung nimmt das Anerbieten mit Dank an und genehmigt sodann den von Grünberg für das betreffende Inserat vorgeschlagenen Wortlaut. - Nachdem noch Soldanski Grülse von Schilsky bestellt und Kuhnt die neu eingegangene Literatur vorgelegt hat, ist der geschäftliche Teil erledigt, und es berichtet zunächst $\mathrm{Kuhnt}$ über ein merkwürdiges Stück einer Leptura-Art, das Rein e ck im Brieselang gefangen und ihm zur Bestimmung übergeben hat. Nach dem ganzen Habitus des Tieres stand es von vornherein fest, dafs es sich nur um eine der 3 Arten: Leptura montana, Leptura dubia oder Leptura sanguinolenta handeln könne. Ferner zeigte die Ausbuchtung des letzten Abdominalsegmentes, dafs das gefangene Tier ein $q$ war. Infolgedessen konnte eine Bestimmung als Leptura montana nicht in Frage kommen, denn das $\mathrm{Kuhnt}$ vorliegende Exemplar zeigte ein schwarzes Halsschild, während bei montana zwar die $\sigma^{\top}$ ein schwarzes, die o dagegen ein rotes Halsschild besitzen. Die beiden übrig bleibenden Arten, dubia und sanguinolenta, haben als gemeinsames Kennzeichen ein schwarzes Halsschild und rote Flügeldecken, die dicht mit Toment besetzt sind. Von letzterem fand sich bei Reinecks Tier keine Spur, die Flügeldecken waren vielmehr vollkommen glatt. Im übrigen war die Behaarung in den Poren schwarz wie bei sanguinolenta, und nicht gelb wie bei dubia. Auch fehlte der für letztere Art charakteristische schwarze Strich an der Seite. $\mathrm{Kuhnt}$ bestimmte also das Tier als eine Varietät von sanguinolenta, die er zu Ehren ihres Entdeckers „varietas Reinecki" nennen wird. - v. Lengerken zeigt darauf unter Bezugnahme auf die von ihm in der D. E. Z. veröffentlichte Arbeit einige Vertreter der Subspezies von Carabus arvensis Hrbst. und macht auf die wesentlichen Unterschiede aufmerksam. - Ullrich erwähnt, dafs v. Lengerken in seiner Arbeit über Car. arvensis angegeben habe, dafs die Tiere der Mark 
Brandenburg gröfser seien als die Vertreter der Subspezies austriae, während Sokolář in der Beschreibung dieser Subspezies besonders betone, dafs sie sich durch die Grölse von den andern unterscheide. v. Lengerken erwidert, dals er aus Posen von Generalmajor Gabriel besonders grofse arvensis germaniae zugleich mit der Mitteilung erhalten habe, dafs der Einsender diese auffallende Grölse der Imagines auf das Folgende zurückführe: die Käfer wären in einem Bestande gefangen, der vorher von der Nonne befallen gewesen sei. Gabriel nehme nun an, dafs die Larven des arvensis in den Raupen der Nonne eine besonders günstige Nahrungsquelle gefunden hätten, und dafs daher die Imagines grölser geworden seien. v. L e n g e r k e $n$ meint, dafs sich durch solche und ähnliche Ursachen das periodische und lokale Auftreten von grölseren Formen, wie in diesem Falle in Posen und in der Mark, ganz gut erklären lasse. - G r ü n be r g legt das neue $\mathrm{Entomologische} \mathrm{Jahrbuch}$ vor und referiert sodann über Alfons Dampf, Zur Kenntnis der gehäusetragenden Lepidopterenlarven, Zool. Jahrb. 1910, eine morphologische Arbeit, zu welcher der Verfasser geführt wurde durch das Bedürfnis, beim Studium der baltischen Bernsteininklusen die rezenten Formen zum Vergleich heranzuziehen. Verfasser untersuchte je 2 Arten aus den Familien der Psychiden, Talaeporiiden und Tineiden und schildert bei jeder Art eingehend die Skeletteile und Beborstung der Kopfkapsel, den Bau der Mundteile, die Sklerite des Körpers und ihre Beborstung. Unter den am Ende der Arbeit zusammengestellten allgemeinen Schlufsfolgerungen ist besonders interessant und wichtig die ganz auffällige Übereinstimmung der Kopf- und Körperborsten nicht nur bei den näher untersuchten, sondern auch bei allen zum Vergleich herangezogenen Arten; sogar bei den Larven der Trichopteren finden sich dieselben Borsten wieder, und es scheint sich die schon von W. M üller geäufserte Ansicht zu bestätigen, dafs wir in den hauptsächlichsten Kopfund Körperborsten eine uralte Einrichtung und ein ganz universelles Merkmal der Lepidopterenlarven zu erblicken haben. Von Wichtigkeit ist auch, dafs bei den untersuchten Arten stets ein kleines Stigma zwischen Meso- und Metathorax nachgewiesen werden konnte, womit verschiedene Beobachtungen älterer Autoren bestätigt werden. Schliefslich geht Verfasser noch auf die mutmafsliche Entstehung der Bauchfülse ein. Die Larve von Adela besitzt am dritten bis sechsten Abdominalsegment an Stelle der Bauchfüfse nur 2 Paar parallele Querreihen kleiner Chitinhäkchen, die sich von den übrigen Härchen des Integuments nur durch ihre stärkere Entwicklung unterscheiden. Der ganze Befund legt die Vermutung nahe, dafs man es hier nicht mit Rudimenten von 
Bauchfüfsen, sondern mit einer phylogenetischen Stufe zu tun hat, aus der wir uns zwanglos durch engeres Zusammentreten der Häkchen und allmähliches Heraustreten aus dem Niveau der Körperoberfläche die Entstehung der Bauchfüfse erklären können. Schlufs 11 Uhr.

Sitzung vom 27. XI. 11. - Beginn 9 Uhr. Die Sitzung leitet der stellvertretende Vorsitzende Gr e in e r. - Das Protokoll der Sitzung vom 20. XI. 11 wird verlesen und genehmigt. Unser Mitglied J. Gi 11 et wohnt jetzt wieder in Nivelles, Belgien, 4 rue de la Religion. - P. Lesne (Asnières) dankt für Aufnahme. - Im Anschlufs an den verlesenen Bericht vom 21. XI. 11 kommt Ulrich noch einmal auf seine Bemerkung über $C$. arvensis zurück und erklärt unter Verlesung zweier Stellen aus der Arbeit des Herrn v. Lengerken, dals ihm die darin enthaltenen Schlüsse, soweit in ihnen der märkische "arvensis“, speziell der Berliner Umgebung, als gröfster Vertreter seiner Art bezeichnet sei, nicht ganz zutreffend schienen. Sämtliche in seinem Besitz befindlichen Stücke, wie auch die ihm aus einigen anderen Sammlungen zu Gesicht gekommenen, seien, im Gegensatz zu dem durch Herrn v. Lengerken vertretenen Standpunkt, kleiner als die bei Wien auftretende austriae-Rasse; aber auch Stücke, die er von Usedom besitzt, desgleichen, laut Mitteilung, auch die der schlesischen Ebene, seien ebenso grols resp. gröfser als Berliner Stücke. Als Beleg zeigt Ulrich 30 von ihm selbst bei Birkenwerder, Stolpe, Finkenkrug und Friedrichshagen erbeutete $C$. arvensis vor, denen zum Vergleich 24 C. arvensis austriae gegenüberstehen, die aus Wien stammen und sich in ihrer Gesamtheit durch ihre Gröfse gut abheben. Ebenso 4 Stücke von Usedom. Ulrich bemerkt noch, dafs die Tiere nicht aus einer gröfseren Masse ausgelesen seien, sondern so, wie er sie erhalten hätte, vorlägen. $\mathrm{K}$ untzen bemerkt zu den Ausführungen des Vorredners, dafs nach dem Material, welches v. Lengerken vorgelegen hat, tatsächlich die zahlreichen märkischen $C$. arvensis germaniae auffallend grofs sind und die durchschnittliche Gröfse tatsächlich die des C. arvensis austriae übertrifft, dafs ferner hinsichtlich der Färbung fast immer die hellbronze Farbe auffällig ist. - An der über die Ansichten von Ulrich und Kuntzen sich anschliefsenden Diskussion beteiligen sich noch $\mathrm{Pape}, \mathrm{R}$ eineck, Ohaus, Böttcher, $\mathrm{Kuhnt}$ und Ahlwarth. - Heyne legt sodann ein Kästchen mit einer Serie vom Hopfenspinner (Hepialus humuli) mit schönen und zum Teil sehr abweichenden Zeichnungen vor. Ferner 4 Cicada septemdecim aus Chicago und 4 ihm unbekannte Dorncicaden, die an einer Akazienart in Pennsylvanien gefunden wurden. An 
Literatur läfst Heyne die Lieferungen des Seitzschen Werkes Nr. 89 der paläarktischen und Nr. 105 und 106 der exotischen Lepidopteren zirkulieren. - $\mathrm{O}$ ha us referiert über 2 kleine Veröffentlichungen unseres Mitgliedes G. A. B a e r in Paris. Die eine behandelt die giftigen Wirkungen des Stiches oder Bisses verschiedener Arthropoden in Peru. Hier konnte B. zunächst die Beobachtung anderer Reisender bestätigen, dafs die von den Eingebornen so sehr gefürchteten Skorpione, Tausendfüfse und Taranteln lange nicht so häufig sind, als die Eingebornen gewöhnlich erzählen, und dafs ferner die Wirkung des Stiches oder Bisses der genannten Tiere stark übertrieben wird. Dagegen sind die Stiche gewisser Hymenopteren, Wespen, Ameisen, und vor allem der Mutillen, ungemein schmerzhaft und verursachen zuweilen schwere Krankheitserscheinungen, Entzündungen, hohes Fieber und Lähmungen. Besonders gefürchtet war in Tocache am Rio Huallaga, dem einen Quellflufs des Amazonas, eine Mutille, Isula genannt; Baer sah nach einem Stich in die Fufssohle bei einer Indianerin Krämpfe, bei einer anderen einen Abort eintreten; nach $\mathrm{T} \mathrm{s} \mathrm{ch} \mathrm{u} \mathrm{di}$ heifst diese Mutille, deren Namen Baer leider nicht angibt, bei den Kitschua-Indianern: Sisi huakan ñahui, Ameise, die einen weinen macht. Die andere Mitteilung betrifft die giftige Wirkung des Stiches eines Ohrwurms, der Apterygida linearis Eschsch. Während seines Aufenthaltes in Santa-Ana, nahe bei Tucuman in Argentinien, im November 1903 war diese Forficulide ungemein häufig und mehrere Bewohner des Hauses wurden von dem Tier gestochen, besonders des Abends im Bett, wenn sie das über das Gesicht oder den Nacken kriechende Insekt entfernen wollten; es liefs sich genau feststellen, dafs das Tier, wenn man es abstreifen wollte, sich mit seinen Zangen einbohrte, um sich festzuhalten. Die Folgen des doppelten Stiches, von dem jedesmal 2 kleine schwarze Punkte zurückblieben, die erst nach längerer Zeit verschwanden, waren Entzündung, Rötung und Schwellung der Umgebung, heftige, brennende Schmerzen und eine weifse harte Quaddel, die erst nach mehreren Tagen wieder verschwand. A hlwarth ladet die Mitglieder zu dem am Donnerstag, den 7. Dezember, stattfindenden Vortrage des Königl. Landesgeologen Herrn Dr. Ka unhowen über "den Untergrund Berlins" im Deutschen Lehrerverein für Naturkunde ein. - Schlufs 11 Uhr.

Sitzung vom 4. XII. 11. - Der Vorsitzende Gr ünberg eröffnet die Sitzung um 9 Uhr. Das Protokoll vom 27. XI. 11 wird genehmigt. - Grünberg macht auf die bevorstehende Generalversammlung am 11. XII. 11 aufmerksam und bittet um 
zahlreichen Besuch. - van L e e u w e n (Semarang - Java) dankt für Aufnahme. - Kuntzen erklärt im Anschlufs an seine Bemerkungen über Carabus arvensis in der vorhergegangenen Sitzung, dafs nach einer Durchsicht des Museumsmaterials wohl die grofsen $C$. arvensis germaniae Lengerk. die Gröfse der grofsen $C$. arvensis austriae Sokolár̆ übertreffen, die durchschnittliche Gröfse der letzteren scheine ihm aber doch bedeutender zu sein als die der märkischen und speziell der Berliner C. arvensis germaniae. - P a p e legt eine für Java neue, äufserst bizarr gebaute Brenthide vor, welche er von unserem Mitgliede Drescher in einem Stück erhielt. Das Tier gehört zur Gattung Calodromus. Aulserdem zeigt er 3 Exemplare von Cyphagogus longulus Senna, gleichfalls aus Java. - O haus legt aus seiner Sammlung ein or der Golofa Championi Bates aus Guatemala vor, das eine eigenartige Verbildung des linken Vorderbeines zeigt. Die Tibia ist kürzer und schmäler als die rechte, und der mittlere Zahn dem Spitzenzahn stark genähert; die Tarsen sind dünn und nur so kurz wie beim + , das Klauenglied nicht verdickt. Das linke Mittel- und Hinterbein ist dagegen ebenso geformt, wie das entsprechende rechte, die Genitalien, d. h. der chitinöse Forceps ganz normal, so dafs die Verbildung des linken Vorderbeines als Hemmungsbildung, wohl infolge einer Verletzung (Bifs) im Larvenstadium, zu betrachten ist. Grünberg bespricht die von H. Konviczka zusammengestellten Etiketten für Käfersammler, die im Verlage der E. Schweizerbartschen Verlagsbuchhandlung in Stuttgart erschienen sind. Ferner referiert Grünberg noch kurz über die Arbeit von R. v. Vofs: Entwicklung von Raupenzeichnung bei einigen Sphingiden. An der daraus entstehenden Diskussion beteiligen sich noch Ulrich, Kuntzen und Soldanski. - Schlufs 11 Uhr.

Aufserordentliche Generalversammlung vom 11. XII. 11. Um 8 Uhr findet eine Vorstandssitzung statt, in welcher verschiedene geschäftliche Angelegenheiten erörtert werden. - Die aufserordentliche Generalversammlung wird vom Vorsitzenden Grünberg um $91 \%$ Uhr eröffnet. Nach Verlesung und Genehmigung des Protokolls der Sitzung vom 4. XII. 11 ergreift Grünberg zu dem schon in unserer Zeitschrift abgedruckten Antrag das Wort und befürwortet das Abhalten gemeinsamer Sitzungen unserer Gesellschaft mit dem Berliner Entomologischen Verein in der Art, dals diese gemeinsamen Sitzungen je einmal im Monat bei jeder der genannten Vereinigungen stattfinden sollen. Redner bemerkt zum Schlufs, dafs der gleiche Antrag vom Berliner Entomologischen Verein bereits mit grofser Majorität 
angenommen wurde. Da für den nunmehr zur Diskussion gestellten Antrag keines von den anwesenden Mitgliedern das Wort wünscht, so wird gleich zur Abstimmung geschritten. Der Antrag wird hierauf mit 22 Stimmen angenommen. - Gr ün berg stellt aufserdem im Namen des gesamten Vorstandes den Antrag, unser um die Gesellschaft so hochverdientes und langjähriges Mitglied J. Schilsky zum Ehrenmitgliede unserer Gesellschaft zu ernennen, welcher Antrag einstimmig zur Annahme gelangt. - Für die Revision der Bibliothek und des Vereinsvermögens werden von Grünberg für die Bibliothek $\mathrm{Pape}$ und $\mathrm{B}$ erka, für das Vereinsvermögen $\mathrm{Sch}$ allehn und Höfig vorgeschlagen, welche das Amt der Revisoren annehmen. Nachdem die Versammlung sich einstimmig für die Wahl der genannten Revisoren erklärt hat, wird die Sitzung gegen $10^{1} / 2$ Uhr geschlossen.

Sitzung vom 18. XII. 11. - Um 8 Uhr findet eine Vorstandssitzung statt. - Die um $9^{1} / 2$ Uhr eröffnete Sitzung leitet der stellvertretende Vorsitzende Greiner. - Das Protokoll der aufserordentlichen Generalversammlung vom 11. XII. 11 wird verlesen und angenommen. - Grünberg teilt mit, dafs er das Resultat der aufserordentlichen Generalversammlung, das Abhalten je einer gemeinsamen Sitzung im Monat, bereits dem Vorsitzenden des Berliner Entomologischen Vereins mitgeteilt und die Mitglieder dieses Vereins zu unserer nächsten Sitzung am 8. I. 12 eingeladen habe. Ferner macht Grünberg noch auf die am 15. I. 12 stattfindende Jahresversammlung aufmerksam. - Kuhnt lälst eine gröfsere Anzahl ihm von verschiedenen Autoren übergebene Separata zirkulieren und bittet um Zusendung weiterer Separata für die Vereinsbibliothek. - Als neues Mitglied wird Herr Paul Mink, Berlin N 65, Ofener Str. 3, vorgeschlagen. - O ha us legt aus seiner Sammlung die auf Ceylon vorkommenden Arten der Rutelidengruppe Adoretini vor, 5 Gattungen mit 10 Arten. Von diesen kommen nur 2 auch aufserhalb Ceylons vor, eine Art, die in dem Ceylon zunächst gelegenen Teil von Vorderindien häufig ist und aufserdem der weit verbreitete Adoretus (Lepadoretus) compressus Weber. Alle anderen Arten sind endemisch und interessant durch ihre Beziehungen zu Madagaskar und Zentral-Afrika. - Zu den Ausführungen von Ohaus bemerkt $\mathrm{Kolbe}$, dafs es sich mit dem Verbreitungsgebiet anderer Coleopterengruppen ähnlich verhält wie bei den Adoretini. An der weiteren Diskussion über dieses Thema beteiligen sich noch Gr ün berg, O ha us, Kuntzen, K o l be, Re in e ck, Höhne, Pape und S o ld anski. — Schlufs 11 Uhr. 


\section{Auszug aus dem Bericht vom 15. I. 12.}

Sitzung vom 15. I. 12. - Beginn $9 \frac{1}{2}$ Uhr. Generalversammlung (Jahresversammlung). - Anwesend 32 Mitglieder. Den Vorsitz führt G r ü n b e r g. -- M o s e r erstattet als Rendant seinen Kassenbericht. Die Revisoren $\mathrm{Höfig}$ und $\mathrm{Schallehn}$ erstatten den Revisionsbericht und beantragen für Moser Entlastung, die erteilt wird. - O ha s berichtet über die Zeitschrift, welche auch im vergangenen Jahr im gleichen Umfang herausgegeben werden konnte. - Kuhnt gibt sodann den Bericht über die Vereinsbibliothek. Pape und Berka berichten über die Revision der Bibliothek und fordern für Kuhnt Entlastung, die erteilt wird. - . Es erfolgt die Neuwahl des Vorstandes durch Zettelwahl, und zwar werden gewählt: Greiner als Vorsitzender, A h lwarth und Gr ün berg als stellvertretende Vorsitzende mit je 30 Stimmen, als Schriftführer K untzen und Berka mit je 28, als Rendant $\mathrm{H}$ öfig mit 30 Stimmen; als Bibliothekar wird $\mathrm{Kuhnt}$ mit 31 , als Redakteur $\mathrm{O}$ h a u s mit 30 Stimmen wiedergewählt. - G r ü n berg dankt dem früheren Vorstande für seine Tätigkeit. - Schlufs $11^{1 / 2}$ Uhr.

\section{Aus der entomologischen Welt.}

\section{Von Hans Soldanski, Wilmersdorf.}

Nachrichten aus unserem Leserkreise, besonders vom Ausland, sind jederzeit willkommen.

Tot en liste.

Der berühmte englische Bergsteiger Edward W h y per, der vielfach auf seinen Reisen auch entomologisch als Sammler tätig war, ist am 16. IX. in Chamounix im Alter von 61 Jahren gestorben. Whymper unternahm am 14. VII. 1865 die erste, zwar erfolgreiche, aber verhängnisvolle Besteigung des Matterhorns, die seinen Gefährten $\mathrm{Huds}$ on, H a d ow und D o u g la s, sowie einem Führer das Leben kostete. Im Jahre 1880 bereiste er Ecuador und bestieg dort - ebenfalls als Erster - beide Gipfel des Chimborazo. Das von ihm über diese Reise veröffentlichte Werk ist betitelt: "Travels amongst the high Andes of the Ecuador" und enthält einen Appendix von $H$. W. B a tes, in welchem die von W. gesammelten Coleopteren behandelt werden. - In Paris stäb J ean Dollfus, der Stifter des Prix Dollfus, den die Entomologische Gesellschaft von Frankreich alljährlich zu vergeben hat. - Rev. Henry C. Mc C o ok in Philadelphia, bekannt durch seine gröfseren Arbeiten über Ernteameisen und über Spinnen, ist 


\section{$2 \mathrm{BHL}$ Biodiversity Heritage Library}

1912. "Aus den Sitzengen." Deutsche entomologische Zeitschrift 1912(1), 83-99. https://doi.org/10.1002/mmnd.48019120108.

View This Item Online: https://www.biodiversitylibrary.org/item/103378

DOI: https://doi.org/10.1002/mmnd.48019120108

Permalink: https://www.biodiversitylibrary.org/partpdf/236104

\section{Holding Institution}

Harvard University, Museum of Comparative Zoology, Ernst Mayr Library

\section{Sponsored by}

Biodiversity Heritage Library

\section{Copyright \& Reuse}

Copyright Status: Public domain. The BHL considers that this work is no longer under copyright protection.

This document was created from content at the Biodiversity Heritage Library, the world's largest open access digital library for biodiversity literature and archives. Visit BHL at https://www.biodiversitylibrary.org. 